\title{
anatomy
}

\section{Partial corpus callosum agenesis and colpocephaly: a case report*}

\author{
Ayşegül Güngör Aydın ${ }^{1}$, Esat Adıgüzel ${ }^{2}$, Aylin Aksoy ${ }^{3}$, Kemalettin Acar ${ }^{4}$ \\ ${ }^{1}$ Department of Neuroscience, Graduate School of Health Sciences, Pamukkale University Denizli, Turkey \\ ${ }^{2}$ Department of Anatomy, School of Medicine, Pamukkale University, Denizli, Turkey \\ ${ }^{3}$ Department of Pathology, Council of Forensic Medicine, Antalya, Turkey \\ ${ }^{4}$ Department of Forensic Medicine, School of Medicine, Pamukkale University, Denizli, Turkey
}

\begin{abstract}
Callosal abnormalities are mostly described as central nervous system malformations and can be symptomatic or asymptomatic. Herein, we report an autopsy case of a 50-year-old Syrian immigrant male with poor academic performance, presented with partial agenesis of the corpus callosum and colpocephaly. He was evidently asymptomatic until death. The cause of death was determined to be hypothermia. Autosy revealed a corpus callosum of $3 \mathrm{~cm}$ only in the anterior part. Posterior part of the corpus callosum, anterior, posterior and hippocampal commissures were absent. On coronal sections, inferior horn of the lateral ventricle was dilated. Histopathological examination showed petechial microhemorrhagic areas and congestion in the brain. This case report was presented to demonstrate a good example of corpus callosum agenesis in asymptomatic individuals except for poor academic performance. Furthermore, this is the first corpus callosum agenesis in medical literature encountered during medicolegal autopsy in an adult case.
\end{abstract}

Keywords: autopsy; corpus callosum agenesis; malformation; poor academic performance; postmortem

Anatomy 2016;10(3):242-245 @2016 Turkish Society of Anatomy and Clinical Anatomy (TSACA)

\section{Introduction}

Corpus callosum is a flat bundle of axonal fibers that connects the two cerebral hemispheres and forms the roof of the lateral ventricles. It is a major midline structure and comprises of approximately 200 million axons. ${ }^{[1]}$ Corpus callosum which is completely formed by $18-20$ th weeks of gestation is one of the latest maturing structures of the brain, and growth is not fully complete until the third decade of life. The formation of sulcus medianus telencephali medii, a median groove in the dorsal lamina reuniens, and massa commissuralis, a large cell mass inside the sulcus medianus telencephali medii formation, seem to be critical steps in the development of the corpus callosum. ${ }^{[2,3]}$ Being exposed to infection, chemical agents and hypoxia during/before or shortly after formation of these structures may likely result in complete or partial agenesis of corpus callosum, respectively. Thus, the portion of corpus callosum present in individuals with callosal abnormalities might be taken as an indication of the time of the insult. ${ }^{[3]}$

Callosal abnormalities are mostly described as central nervous system malformations and can be symptomatic or asymptomatic. The overall incidence of central nervous system malformations is about 1 in 100 births. ${ }^{[4]}$ The prevalence of corpus callosum agenesis in the general population is approximately 7 per 1000, but 3 per 100 in the developmentally delayed population. Because callosal agenesis is often asymptomatic, the actual prevalence of corpus callosum agenesis is not clear. ${ }^{[5,6]}$

Most cases of abnormal corpus callosum were associated with other central nervous system structural abnor-

This work has been presented at the XIV Turkish Neuroscience Congress, May 26-29, 2016, Ankara, Turkey. 
malities like cortical dysplasia, dilatation of the occipital horns of the lateral ventricles (colpocephaly), and white matter fibers that developmentally curl back and fail to bridge the two hemispheres (Probst bundles). In addition to these structural anomalies, corpus callosum agenesis is commonly accompanied by other neurodevelopmental abnormalities such as reduced extracallosal white matter volume, abnormalities of the cerebral ventricles, hippocampal commissure, anterior commissure, cerebellar vermis, cerebellar hemispheres and brainstem. ${ }^{[7,8]}$

We presented here a case of a man with corpus callosum agenesis who was evidently asymptomatic until death. This case was encountered during routine medicolegal autopsy. He was a Syrian immigrant, there was no information for his medical history. Our limited knowledge about the case was that he had poor academic performance and he was known as gullible, but was able to continue his daily life. This case report was presented to demonstrate a good example of corpus callosum agenesis in asymptomatic individuals except poor academic performance. We discuss the postmortem findings in the light of current knowledge of functional outcomes in individuals with corpus callosum agenesis.

\section{Case Report}

The case was a 50-year-old Syrian immigrant male whose medical history was unknown. His body was found in his room by his brother. A medicolegal autopsy was performed according to routine protocols. He was $75 \mathrm{~kg}$ in weight and $165 \mathrm{~cm}$ in height, and his appearance was consistent with his stated age. The autopsy revealed no signs related to violent or traumatic cause of death. The postmortem interval was estimated as twelve hours. Brain and brainstem were removed by using standard autopsy techniques and weighed as $1465 \mathrm{~g}$. There were no unusual findings at the skull base. The cerebral hemispheres were symmetrical. The dura and leptomeninges were unremarkable. The morphological examination of brain showed mild congestion and venous distension. The surface of the occipital lob was collapsed because of colpocephaly. The circle of Willis was usual. The olfactory bulbs and tracts were normal. The brainstem was separated from the cerebral hemispheres by a section at the level of the midbrain. The cerebral hemispheres were bisected along the mid-sagittal plane. The corpus callosum was inspected and photographed at the interhemispheric aspect. The corpus callosum of $3 \mathrm{~cm}$ was present only in the anterior part. Posterior part of the corpus callosum, anterior, posterior and hippocampal commissures were absent (Figure 1). Two cerebral hemispheres were adherent in the lower part of the medial surface of the frontal lobe. There was no cingulate gyrus, and gyri of temporal lobe run vertically to diencephalon in the medial surface. There were no interhemispheric cysts or tumors. Serial sectioning was performed in the coronal plane at $2 \mathrm{~cm}$ interval from the frontal to the occipital poles. Each slice was photographed separately for further evaluation. On coronal sections, inferior horn of the lateral ventricle was
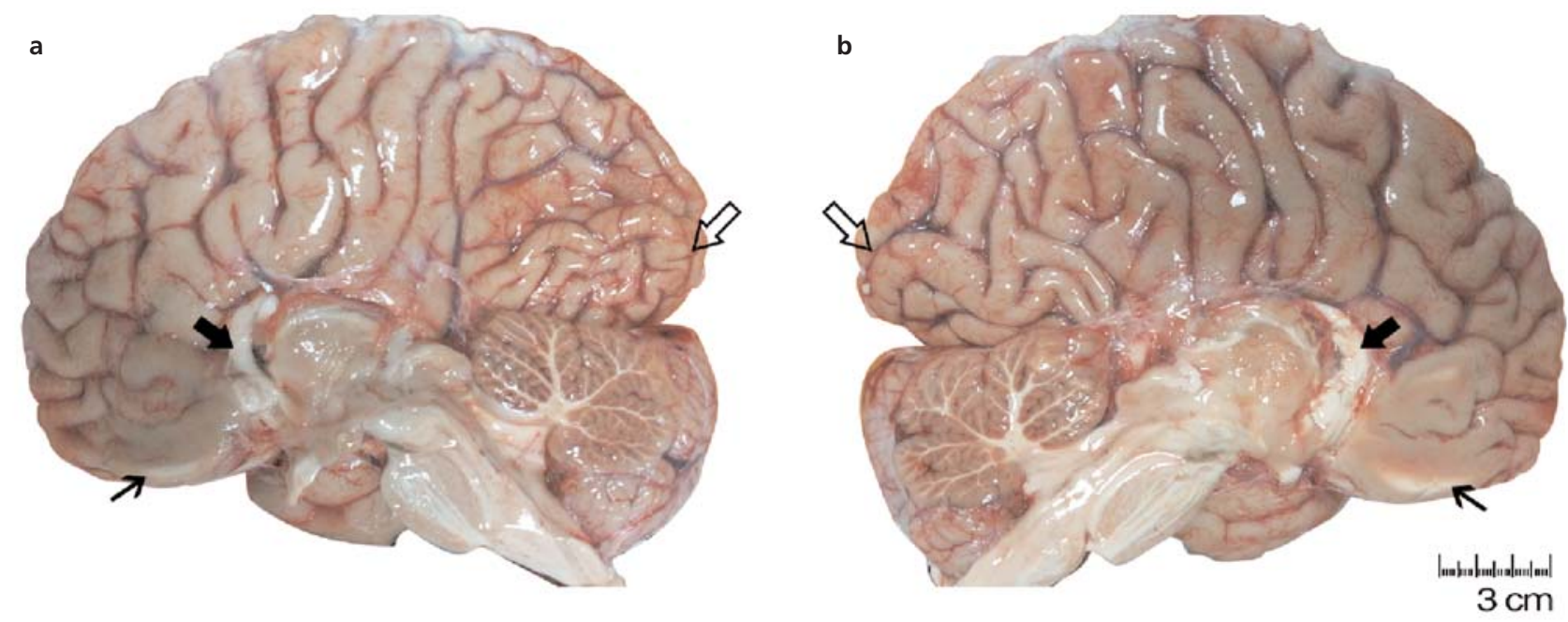

Figure 1. Mid-sagittal aspect of cerebral hemispheres of the case with partial agenesis of corpus callosum. Right cerebral hemisphere (a), left cerebral hemisphere (b). The corpus callosum of $3 \mathrm{~cm}$ was present in the anterior part (thick arrows). Posterior part of corpus callosum, anterior, posterior and hippocampal commissures were absent. Two cerebral hemispheres were adherent in the lower part of the medial surface of the frontal lobe (thin arrows). The surface of occipital lobes were collapsed because of colpocephaly (open arrows). [Color figure can be viewed in the online issue, which is available at www.anatomy. org.tr] 
dilated and colpocephaly was determined (Figure 2). Left pole of temporal lobe had small cortical lytic-necrotic areas and was disrupted during the dissection. Occipital lobe was thinner than the other lobes. Cerebellum was sectioned along the sagittal plane. The cerebellar folia and dentate nuclei were unremarkable. The aqueduct and the fourth ventricle were of normal configuration, and the spinal cord was normal. Results of routine postmortem toxicological analysis were unremarkable. This case report complies with the current laws of the country (Turkey) in which the study was performed.

\section{Histopathological examination}

Routine medicolegal histopathologic examination showed petechial microhemorrhagic areas and congestion in the brain and cerebellum, autolytic changes and areas of focal fat necrosis and fresh microhemorrhage in parenchyma and peripheral adipose tissue of pancreas, autolytic changes in superficial epithelium, mild moderate chronic gastritis, multifocal fresh microhemorrhagic areas, swelling of epithelial cells and vacuolization in focal areas, extremely dilatation and severe congestion in submucosal vessels in the stomach. Considering the findings at autopsy and histopathologic examination, the cause of death was determined to be hypothermia.

\section{Discussion}

This is a case study of a 50-year-old man who died due to hypothermia and presented with partial agenesis of the corpus callosum and colpocephaly. Regarding information given by his relative, he could continue his daily life but he had poor academic performance. He immigrated from Syria and was living in poor conditions and poverty recently.

Most of the prevelance studies about corpus callosum agenesis were performed using neuroimaging techniques such as ultrasound and magnetic resonance imaging, especially during the prenatal and postnatal periods. ${ }^{[9,10]}$

Recent neonatal and prenatal imaging studies suggest that corpus callosum agenesis occurs in at least 1:4,000 live births, ${ }^{[1]]}$ and other imaging studies demonstrated that $3-5 \%$ of the individuals assessed for neurodevelopmental disorders have corpus callosum agenesis. ${ }^{[12]}$

In a prevelance study by Kidron et al. ${ }^{[13]}$ determined four distinct callosal defect groups which are complete (absence of all components), partial (presence of a short remnant), hypoplastic (thinning of the all parts of the corpus callosum), and mixed defects (partial corpus callosum agenesis with thinning of the residual part of the corpus callosum). However, regardless of corpus callosum morphology, Brescian et al. ${ }^{[7]}$ classified agenesis of corpus cal-

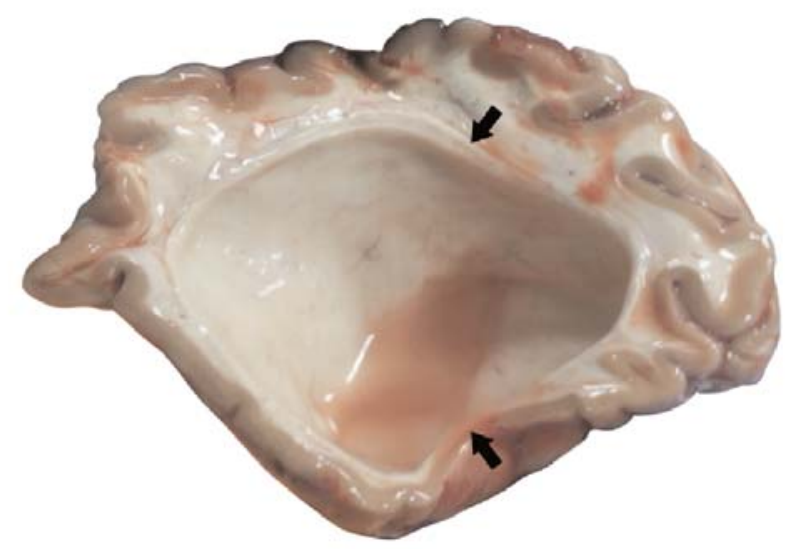

Figure 2. Coronal section through occipital lobe showing disproportionate enlargement of the occipital horn of the lateral ventricle (colpocephaly) (black arrows indicate the cut edge of occipital horn of the lateral ventricle). [Color figure can be viewed in the online issue, which is available at www.anatomy. org.tr]

losum in two groups according to the presence of Probst bundles. In Type 1, primary agenesis of corpus callosum, Probst bundles are present and along with the enlargement of the occipital horns of the lateral ventricles (colpocephaly). Conversely, in Type 2, Probst bundles and commissural axons are absent. ${ }^{[7]}$ Some researchers also classified agenesis of corpus callosum with the presence of other cerebral or systemic abnormalities. In this classification, "isolated type" is without any other abnormalities, while "complex type" is concomitant with cerebral or extracerebral abnormalities such as holoprosencephaly, cyst formation, metabolic diseases. ${ }^{[14,15]}$

Taking into consideration the mentioned classifications, this case is a partial agenesis according to Kidron classification, and presence of colpocephaly is appropriate with Type 1 of Brescian classification, but without magnetic resonance imaging it was not possible to evaluate Probst bundles. ${ }^{[7,13]}$ Lastly, it may be considered as complex type of corpus callosum agenesis, due to accompanying poor academic performance. Comparing this case with the classification studies based on the fetal period may be controversial, because this is an adult case.

It is reported that the majority of cases with isolated agenesis of the corpus callosum show no symptoms. A systematic review of the literature recently suggested that about $65 \%$ of cases with complete agenesis of the corpus callosum had normal intelligence and neurodevelopment at postnatal period. ${ }^{[14]}$ Cases with corpus callosum agenesis may accompany other anomalies without showing any symptoms,; therefore, it should be considered that these anomalies may not only be related to corpus callosum agenesis. 
Our case report had several limitations. First of all, because it was a medicolegal autopsy case, we had limited time to perform detailed postmortem anatomical dissection, we could not keep the whole brain tissue. Furthermore, we did not have enough information about the medical history of the case; also, we could not confirm the information received from the relatives. On the other hand, we could take postmortem standard histopathological and toxicological examination reports.

\section{Conclusion}

This is the first corpus callosum agenesis in medical literature encountered during medicolegal autopsy in an adult. The authors emphasize that the clinical anatomy and forensic medicine cooperation may contribute significantly to medical literature. In conclusion, this case underlines the need for increased awareness of corpus callosum agenesis from the point of clinical findings. Also, corpus callosum agenesis should be considered in individuals with poor academic performance. Neuroimaging techniques can be useful to identify the causes of poor academic performance.

\section{Acknowledgments}

The authors thank to Gökcen Köseler for helping the preparation of the figures.

\section{References}

1. Aboitiz F, Montiel J. One hundred million years of interhemispheric communication: the history of the corpus callosum. Braz J Med Biol Res 2003;36:409-20.

2. Ramaekers G. Embryology and anatomy of the corpus callosum. In: Ramaekers G, Njiokiktjien C, editor. Pediatric behavioural neurology: the child's corpus callosum. Vol 3. Amsterdam: Suyi Publications; 1991. p. 24-39.

3. Barkovich AJ, Norman D. Anomalies of the corpus callosum: Correlation with further anomalies of the brain. AJR Am J Roentgenol $1988 ; 151: 171-9$
4. Gianluigi P, McGahan JP, Nyberg DA. Cerebral malformations. In: Nyberg DA, McGahan JP, Pretorius DH, Pilu G, editors. Diagnostic imaging of fetal anomalies. Philadelphia (PA): Lippincott Williams and Wilkins; 2002. p. 221-91.

5. Derbali F, Ben FF, Rezgui A, Karmani M, Hajji R, Ben AO, Mzabi A, Laouani C. The corpus callosum agenesis: a case report. American Journal of Medical Case Reports2015;3:27-29.

6. Jeret JS, Serur D, Wisniewski K, Fisch C. Frequency of agenesis of the corpus callosum in the developmentally disabled population as determined by computerized tomography. Pediatr Neurosci 1985 ; 12:101-3.

7. Brescian NE, Curiel RE, Gass CS. Case study: a patient with agenesis of the corpus callosum with minimal associated neuropsychological impairment. Neurocase 2013;20:606-14.

8. Hetts SW, Sherr S, Chao S, Gobuty S, Barkovich AJ. Anomalies of the corpus callosum: An MR analysis of the phenotypic spectrum of associated malformations. AJR Am J Roentgenol 2006;187:1343-8.

9. Contro E, Nanni M, Bellussi F, Salsi G, Grisolia G, Sanz-Cortès M, Righini A, Rizzo N, Pilu G, Ghi T. The hippocampal commissure: a new finding at prenatal 3D ultrasound in fetuses with isolated complete agenesis of the corpus callosum. Prenat Diagn 2015;35:91922.

10. Tang PH, Bartha AI, Norton ME, Barkovich AJ, Sherr EH, Glenn OA. Agenesis of the corpus callosum: an MR imaging analysis of associated abnormalities in the fetus. AJNR Am J Neuroradiol 2009; 30:257-63

11. Glass H, Shaw GM, Ma C, Sherr EH. Agenesis of the corpus callosum in California 1983-2003: a population-based study. Am J Med Genet A 2008;146A:2495-500.

12. Bodensteiner J, Schaefer GB, Breeding L, Cowan L. Hypoplasia of the corpus callosum: a study of 445 consecutive MRI scans. J Child Neurol 1994;9:47-9.

13. Kidron D, Shapira D, Ben SL, Malinger G, Lev D, Cioca A, Sharony R, Lerman ST. Agenesis of the corpus callosum. An autopsy study in fetuses. Virchows Arch 2016;468:219-30.

14. Santo S, D'Antonio F, Homfray T, Rich P, Pilu G, Bhide A, Thilaganathan B, PapageorghiouAT. Counseling in fetal medicine: agenesis of the corpus callosum. Ultrasound Obstet Gynecol 2012; 40:513-21.

15. Guillermo Davila-Gutierrez. Agenesis and dysgenesis of the corpus callosum. Semin Pediatr Neurol 2002;9:292-301.

Correspondence to: Esat Adıgüzel, MD

Department of Anatomy, School of Medicine,

Pamukkale University, Denizli, Turkey

Phone: +905427118031

e-mail: adiguzel@pau.edu.tr

Conflict of interest statement: No conflicts declared.

This is an open access article distributed under the terms of the Creative Commons Attribution-NonCommercial-NoDerivs 3.0 Unported (CC BY-NCND3.0) Licence (http://creativecommons.org/licenses/by-nc-nd/3.0/) which permits unrestricted noncommercial use, distribution, and reproduction in any medium, provided the original work is properly cited. Please cite this article as: Güngör Aydın A, Adıgüzel E, Aksoy A, Acar K. Partial corpus callosum agenesis and colpocephaly: a case report. Anatomy 2016;10(3):242-245. 